\title{
Contribuciones en la modelación de energía renovable en sistemas de potencia
}

Fecha de recepción: 16 de febrero de 2018

Fecha de aprobación: 24 de octubre de 2018

\author{
Sergio Raúl Rivera-Rodríguez ${ }^{1}$ \\ Universidad Nacional de Colombia - Sede Bogotá \\ srriverar@unal.edu.co \\ Sergio Andrés Dorado-Rojas \\ Universidad Nacional de Colombia - Sede Bogotá \\ sadorador@unal.edu.co
}

DOI: https://doi.org/10.21158/23823399.v6.n0.2018.2362

Cómo citar este artículo: Rivera-Rodríguez, S. R.; Dorado-Rojas, S. A. (2018). Contribuciones en la modelación de energía renovable en sistemas de potencia. Revista Ontare, 6, 7-34. DOI: https://doi. org/10.21158/23823399.v6.n0.2018.2362

\section{Resumen}

En este artículo se realiza un análisis de la literatura científica producida en materia de modelación y simulación de sistemas de potencia con penetración de energía renovable. Por medio de una revisión documental de los trabajos publicados por el investigador en cuestión, se sintetizaron los aspectos más innovadores de tales estudios y las herramientas utilizadas, con el fin de aportar una valiosa contribución a la búsqueda de soluciones óptimas de los problemas operacionales y de planeación que se presentan en los sistemas eléctricos gracias a la creciente penetración de fuentes de energía renovable.

Dicha revisión se abordó a través de la agrupación del material científico de acuerdo con las siguientes subtemáticas: energía renovable en Latinoamérica y Colombia; modelación de incertidumbre de sistemas solares, eólicos, pequeñas centrales hidroeléctricas y vehículos eléctricos; y optimización heurística aplicada a sistemas de potencia con penetración de energía renovable.

1 Ingeniero electricista (grado de honor) y estudiante de la Maestría en Ingeniería-Automatización Industrial de la Universidad Nacional de Colombia. 
A partir de ello se exponen tanto la aplicabilidad como los beneficios del desarrollo de formulaciones matemáticas, por lo general complejas, que permiten garantizar robustez, estabilidad, eficiencia, flexibilidad y rentabilidad de dichos sistemas.

Como resultado de la aplicación de la modelación de incertidumbre propuesta en redes similares a las identificadas, se obtuvo la compilación simplificada de un bagaje invaluable de conocimientos particularmente útiles para evaluar la integración de las tecnologías de las energías renovables en cualquier sector económico.

\section{Palabras clave}

Energía renovable; sistemas de energía renovable; modelación de sistemas; simulación de sistemas; sistemas de potencia; estado del arte. 


\title{
Contributions to the modeling of renewable energy in power systems
}

\begin{abstract}
This article analyzes the scientific literature that has been produced on the modeling and simulation of power systems with renewable energy penetration. It is by means of a documentary review of the works that have been published by the researcher in question that we synthesized the most innovative aspects of such studies and the tools that were used, in order to make a valuable contribution to the search for optimal solutions to operational and planning problems that can be seen in electrical systems due to the increasing penetration of renewable energy sources.
\end{abstract}

This review was addressed through the grouping of scientific material according to the following sub-themes: renewable energy in Latin America and Colombia; uncertainty modeling of solar and wind systems, small hydroelectric plants, and electric vehicles; and heuristic optimization applied to power systems with renewable energy penetration.

From this, we exposed both the applicability and the benefits of the development of mathematical formulations, usually complex, which guarantee the robustness, stability, efficiency, flexibility, and profitability of these systems.

As a result of the application of the uncertainty modeling that was proposed in networks similar to those that were identified, we obtained the simplified compilation of an invaluable baggage of particularly useful knowledge to evaluate the integration of renewable energy technologies in any economic sector.

\section{Keywords:}

Renewable energy; renewable energy systems; systems modeling; systems simulation, power systems; state of the art. 


\title{
Contribuições na modelagem de energia renovável em sistemas de energia
}

\begin{abstract}
Resumo
Este artigo analisa a literatura científica produzida sobre modelagem e simulação de sistemas de energia de potência com penetração de energia renovável. Por meio de uma revisão documental dos trabalhos publicados pelo pesquisador em questão, foram sintetizados os aspectos mais inovadores de tais estudos e as ferramentas utilizadas, a fim de dar uma contribuição valiosa para a busca de soluções ótimas para problemas operacionais e planejamento apresentado em sistemas elétricos, graças à crescente penetração de fontes de energia renováveis. Esta revisão foi abordada por meio do agrupamento de material científico de acordo com os seguintes subtemas: energia renovável na América Latina e Colômbia; Modelagem de incertezas de sistemas solares, eólicos, pequenas usinas hidrelétricas e veículos elétricos e otimização heurística aplicada a sistemas de energia com penetração de energia renovável.

A partir disso, são expostas a aplicabilidade e os beneficios do desenvolvimento de formulações matemáticas, geralmente complexas, que garantem robustez, estabilidade, eficiência, flexibilidade e rentabilidade desses sistemas.

Como resultado da aplicação da modelagem de incerteza proposta em redes semelhantes às identificadas, foi obtida a compilação simplificada de uma bagagem inestimável de conhecimento particularmente útil para avaliar a integração de tecnologias de energia renovável em qualquer setor econômico.
\end{abstract}

\section{Palavras-chave}

Energia renovável; sistemas de energia renovável; modelagem de sistemas; simulação de sistemas; sistemas de energia; estado da arte. 


\section{Contributions à la modélisation des énergies renouvelables dans les systèmes de production énergétique}

\section{Résumé}

Cet article analyse la littérature scientifique évoquant la modélisation et la simulation de systèmes de production énergétique avec utilisation d'énergies renouvelables. Une synthèse de ces travaux, publiés par des scientifiques, révèle les aspects les plus novateurs de ces études, et les outils utilisés contribuant à la recherche de solutions optimales aux problèmes opérationnels de planifications répertoriés dans les systèmes énergétiques et ce, grâce à une utilisation croissante des sources d'énergie renouvelables.

Cet article contient du matériel scientifique des champs d'application suivants: énergies renouvelables en Amérique latine et en Colombie ; modélisations de l'incertitude énergétique des centrales solaires et éoliennes, petites centrales hydroélectriques et véhicules électriques et, une optimisation heuristique appliquée aux systèmes énergétiques utilisant des énergies renouvelables.

Une déduction de l'applicabilité et des avantages d'un développement de formulations mathématiques complexes, garantissant la robustesse, la stabilité, l'efficacité, la flexibilité et la rentabilité de ces systèmes a ainsi pu être réalisée.

Enfin, suite à l'application de la modélisation de l'incertitude à des réseaux similaires à ceux identifiés, il a été possible d'obtenir une compilation simplifiée de connaissances particulièrement utiles pour évaluer la factibilité de l'intégration des technologies en énergies renouvelables dans différents secteurs économiques.

\section{Mots-clés}

Énergie renouvelable; systèmes d'énergie renouvelable; modélisation des systèmes; simulation de systèmes; systèmes énergétiques; état de l'art. 


\section{Introducción}

T $\mathrm{n}$ los sistemas de potencia contemporáneos se promueve, desde hace Calgún tiempo, el uso de energías renovables con el fin de reducir las emisiones que surgen de la generación de energía con plantas a base de hidrocarburos (Arango, Urrego y Rivera, 2017). Gracias a esto, los operadores del sistema requieren de herramientas de análisis que les permitan incorporar estas nuevas formas de energía.

El presente artículo pretende, a través de una revisión documental propia, compilar de una manera sistemática los aspectos más importantes de las investigaciones realizadas por el autor en esta materia. Los aportes presentados resaltan la significancia del empleo de metodologías innovadoras para la toma de decisiones por parte de los operadores de los sistemas de potencia. Las herramientas propuestas consideran las incertidumbres de las fuentes primarias de la energía renovable, con lo cual se demuestra alta aplicabilidad y beneficios operacionales.

La descripción de la propuesta se aborda a través de la agrupación del material científico del autor, de acuerdo con las siguientes subtemáticas:

- Subtemática I. Energía renovable en Latinoamérica y Colombia; presenta la motivación y la necesidad del presente estudio.

- Subtemática II. Modelación de incertidumbre de sistemas solares, eólicos, pequeñas centrales hidroeléctricas y vehículos eléctricos; desarrolla algunos de los objetivos del presente estudio, correspondientes a las herramientas a utilizar para considerar las incertidumbres de las fuentes primarias.

- Subtemática III. Optimización heurística aplicada a sistemas de potencia con penetración de energía renovable; desarrolla el objetivo de proponer soluciones de operación, planeación y modelación de sistemas de potencia con energías renovables. 
La hipótesis utilizada es que mediante herramientas de modelación de incertidumbre y optimización, es posible abordar los problemas de las redes actuales y futuras de potencia. La metodología corresponde a la presentación de los aportes obtenidos en las últimas publicaciones del autor - veintidós trabajos publicados y aceptados- La contribución del presente trabajo se refiere a la exposición académica de las herramientas propuestas por el autor, a fin de que los diferentes agentes de generación, transmisión, distribución y comercialización se apropien de los métodos de modelación y optimización en la simulación de sistemas de potencia con renovables.

Figura 1. Aportes en la modelación y la simulación de sistemas de potencia con penetración de energía renovable

\section{Modelación y simulación de sistemas de potencia con penetración de renovables}

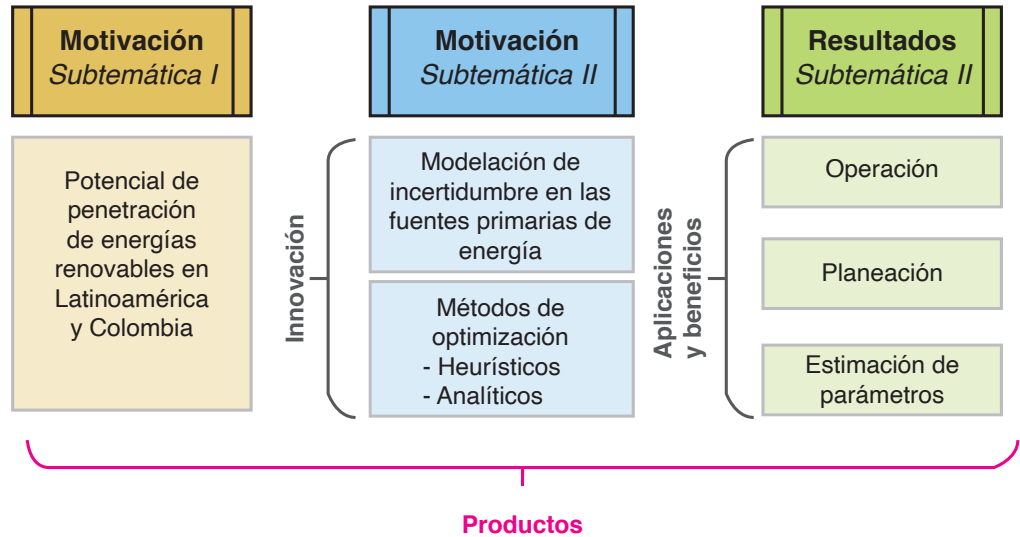

22 Publicaciones

Diseño de competencia internacional de optimización con operación de sistemas con renovables

Fuente. Elaboración propia. 


\section{Presentación del problema y del objetivo}

7 n sistemas eléctricos de potencia, cuando se incorporan tecnologías que utilizan recursos renovables (Valencia-Zuluaga, Rivera y Sánchez-Ortiz, 2017), como, por ejemplo, la generación fotovoltaica, la generación con energía eólica, o cargas controlables tales como vehículos eléctricos, se introduce cierta incertidumbre asociada a la disponibilidad de potencia en el sistema que es consecuencia de la estocasticidad de los recursos energéticos primarios (Arévalo, Santos y Rivera, 2017b).

Tal incertidumbre es indeseada en la programación del despacho de energía y se considera uno de los obstáculos que ha impedido la alta penetración de los recursos renovables en los sistemas de generación. Debido a esto, el principal aporte de este artículo es analizar el trabajo realizado por el autor en lo que concierne a los costos de incertidumbre, una manera vanguardista de incorporar los modelos probabilísticos que describen las fuentes primarias de generación renovable dentro del problema clásico de optimización del despacho económico de carga. Estos costos constituyen herramientas de planeación que brindan una solución económicamente viable en el despacho económico, sin afectar la disponibilidad de potencia en la demanda (Arévalo, Santos y Rivera, 2017a).

En el propósito de abordar esta problemática se propone, en primer lugar, identificar plenamente la motivación y la necesidad del presente estudio - Subtemática I- Enseguida se describe la herramienta más adecuada de modelación de incertidumbre — Subtemática II- Finalmente, se presentan los resultados del uso de esta herramienta en la operación, la planeación y la modelación de redes de potencia con energías renovables — Subtemática III- El presente artículo busca sintetizar de manera cualitativa los aportes del autor en estas direcciones de investigación, definidas por las tres subtemáticas mencionadas. 


\section{Descripción de la propuesta}

7 ste estudio documental procura exponer los avances en materia de modelación y simulación de sistemas de alta penetración de energía renovable, mediante la revisión sistemática de literatura científica referida a las investigaciones del autor sobre el tema en el último año — agosto 2016-agosto 2017- A continuación, se sintetizan los aspectos más importantes de los artículos de mayor relevancia que sustentan la propuesta, al abordar el problema mediante su división en subtemáticas.

Las subtemáticas I y II — presentadas en esta sección — se refieren a la motivación y la necesidad de este estudio, específicamente en Latinoamérica y Colombia, y la propuesta de modelación al considerar incertidumbres en los recursos primarios de la energía renovable.

\subsection{Subtemática I: energía renovable en Latinoamérica y Colombia}

\subsubsection{Microrredes en países en desarrollo.}

Se consideran países en desarrollo aquellos cuyas economías se encuentran en proceso de crecimiento económico a partir del subdesarrollo o de una economía de transición (Rivera, Ñustes, León y Rodríguez, 2017). En específico, dos tercios de la población mundial vive en países en desarrollo ubicados, principalmente, en América Latina, África y Asia (Rivera et al., 2017). Este último artículo describe la infraestructura eléctrica en estos territorios, así como expone sus características, fortalezas, debilidades y oportunidades.

Como elemento de particular interés se afirma que las fuentes de energía renovables se utilizan de manera amplia en el sector eléctrico de América Latina, y también en el Caribe (Tabla 1), donde existe un gran potencial en energía hidroeléctrica, geotérmica, biomasa, solar y eólica; 
son estas fuentes primarias de las que se puede hacer uso de forma ilimitada (Rivera et al., 2017).

De igual forma, se detallan las especificaciones generales asociadas a las investigaciones y los avances en la utilización de microrredes y en la generación distribuida, así como el modelado de sistemas que involucran diferentes fuentes renovables y estrategias de control aplicadas a diversos escenarios. Concluye con la presentación de las normas y las políticas sobre la energía limpia en países en desarrollo, junto con una descripción detallada de los proyectos en microrredes que se desarrollan en la actualidad. Adicionalmente, el autor realizó un estudio similar de microrredes en Europa y Estados Unidos (Rivera y León, 2017; Rivera y ValenciaZuluaga, 2017), en el cual identifica las diferencias de las redes en estas zonas con respecto a las de los países en desarrollo.

\subsubsection{Mercados de energía en América Latina: oportunidades de inversión en fuentes renovables no convencionales.}

A través de esta publicación se exhiben las potencialidades de América Latina para la introducción de fuentes de energía renovable no convencionales (FERNC) como soluciones energéticas, gracias a su extensa riqueza, desde la perspectiva de la diversidad de fuentes de energía, con abundancia de generación hidroeléctrica, eólica y solar (Vargas, Saavedra, Samper, Rivera y Rodríguez, 2016).

Así mismo, se describe la evolución de la generación y las regulaciones de las FERNC, los progresos en la implementación de generación distribuida y redes de distribución inteligentes, la asignación de los costos de las redes de transmisión, los retos y las oportunidades de inversión para la energía renovable en América Central, Chile, Brasil, Uruguay, Colombia y Argentina. 
Tabla 1. Algunos indicadores de la penetración de generación de renovables en Latinoamérica y el Caribe

\begin{tabular}{|c|c|c|c|}
\hline & $\begin{array}{c}\text { Capacidad instalada en } \\
\text { renovables }\end{array}$ & $\begin{array}{l}\text { Inversión en } \\
\text { microrredes y } \\
\text { renovables } \\
\text { (\% del PIB) }\end{array}$ & Tecnologías \\
\hline Colombia & $\begin{array}{c}68,5 \% \\
(63,70 \% \mathrm{H}+3,80 \% \mathrm{~W}+ \\
1,00 \% \mathrm{PV})\end{array}$ & -- & $\begin{array}{l}\text { Hidráulica (H), eólica } \\
\text { (W) y solar (PV) }\end{array}$ \\
\hline Argentina & $\begin{array}{c}27,4 \% \\
(27 \% H+0,36 \% W+ \\
0,04 \% P V)\end{array}$ & $0,58 \%$ & $\begin{array}{l}\text { Eólica }(W) \text {, solar } \\
\text { fotovoltaica }(P V) \text {, } \\
\text { hidrocinética }(H)\end{array}$ \\
\hline Bolivia & $\begin{array}{c}18,7 \% \\
(15,21 \% \mathrm{H}+3,49 \% \mathrm{PV})\end{array}$ & -- & $\begin{array}{l}\text { Hidroeléctrica }(\mathrm{H}) \text { y } \\
\text { solar }(\mathrm{PV})\end{array}$ \\
\hline Brasil & $\begin{array}{c}32 \% \\
(28 \% H+3,00 \% W+ \\
1,00 \% P V)\end{array}$ & $1,15 \%$ & $\begin{array}{l}\text { Hidro- y turbogene- } \\
\text { radores }(H) \text {, turbinas } \\
\text { eólicas }(\mathrm{W}) \text {, solar } \\
\text { fotovoltaica }(\mathrm{PV})\end{array}$ \\
\hline Chile & $\begin{array}{c}52 \% \\
(40 \% \mathrm{H}+10 \% \mathrm{~W}+ \\
2,00 \% \mathrm{PV})\end{array}$ & $0,36 \%$ & $\begin{array}{l}\text { Hidráulica (H), eólica } \\
\text { (W) y solar (PV) }\end{array}$ \\
\hline Cuba & -- & -- & -- \\
\hline Ecuador & $\begin{array}{c}54 \% \\
(49 \% \mathrm{H}+4,00 \% \mathrm{~W}+ \\
1,00 \% \mathrm{PV})\end{array}$ & -- & $\begin{array}{l}\text { Hidráulica (H), eólica } \\
\text { (W) y solar (PV) }\end{array}$ \\
\hline Guatemala & $\begin{array}{c}52 \% \\
(49 \% \mathrm{H}+2,50 \% \mathrm{~W}+ \\
0,50 \% \mathrm{PV})\end{array}$ & -- & $\begin{array}{l}\text { Hidráulica (H), eólica } \\
\text { (W) y solar (PV) }\end{array}$ \\
\hline México & $\begin{array}{c}13,90 \% \\
(13,60 \% \mathrm{H}+0,10 \% \mathrm{~W}+ \\
0,20 \% \mathrm{PV})\end{array}$ & $0,50 \%$ & $\begin{array}{l}\text { Hidráulica (H), eólica } \\
\text { (W) y solar (PV) }\end{array}$ \\
\hline Paraguay & -- & -- & -- \\
\hline Perú & $\begin{array}{c}53 \% \\
(46 \% \mathrm{H}+4,00 \% \mathrm{~W}+ \\
3,00 \% \mathrm{PV})\end{array}$ & & $\begin{array}{l}\text { Hidráulica (H), eólica } \\
\text { (W) y solar (PV) }\end{array}$ \\
\hline
\end{tabular}




\begin{tabular}{|l|c|l|l|}
\hline Uruguay & $\begin{array}{c}54,4 \% \\
\left(\begin{array}{c}4,36 \%+12,93 \% \mathrm{~W}+ \\
0,11 \% \mathrm{PV})\end{array}\right.\end{array}$ & $\begin{array}{c}\text { Hidráulica }(\mathrm{H}) \text {, eólica } \\
(\mathrm{W}) \text { y solar }(\mathrm{PV})\end{array}$ \\
\hline Venezuela & $\begin{array}{c}71 \% \\
\left(\begin{array}{c}69 \mathrm{H}+1,60 \% \mathrm{~W}+ \\
0,40 \% \mathrm{PV})\end{array}\right.\end{array}$ & $\begin{array}{c}\text { Hidráulica }(\mathrm{H}) \text {, eólica } \\
\text { (W) y solar (PV) }\end{array}$ \\
\hline
\end{tabular}

Fuente. Rivera et al., 2017.

Finalmente, se asevera que América Latina debe considerarse un territorio prometedor para la penetración de FERNC, gracias al déficit y la incertidumbre en la generación energética que enfrentan algunos países, evidenciándose, en consecuencia, atractivas oportunidades comerciales en esta área.

\subsubsection{Colombia: territorio de inversión en fuentes no convencionales de energía renovable para la generación eléctrica.}

La descripción del panorama del sector eléctrico colombiano revela la gran debilidad de la estructura actual de la matriz de generación eléctrica del país, de modo que demuestra la baja confiabilidad del sistema en cuanto al suministro eléctrico, gracias a la incertidumbre del recurso hidroeléctrico y su vulnerabilidad (Ñustes y Rivera, 2017).

Al considerar este aspecto, Ñustes y Rivera (2017) analizan la evolución de la industria eléctrica colombiana en las últimas décadas y exponen la problemática actual, en adición a la visión de los diferentes agentes del sector eléctrico. El contexto muestra la oportunidad de inversión para diversos actores en generación distribuida, redes inteligentes, microrredes y parques de generación renovable no tradicionales en cuanto forma de afrontar las problemáticas señaladas (Vargas et al., 2016), tales como la complementariedad de distintas fuentes de generación renovables no tradicionales con las tradicionales hidroeléctricas, la reducción de la vulnerabilidad de los grandes consumidores a las fluctuaciones de los precios y el suministro de menores costos y mayor confiabilidad en las zonas no interconectadas (ZNI). 
Se culmina con la presentación de la disposición de fuentes de financiamiento a nivel nacional e internacional para la puesta en marcha de proyectos de generación distribuida con fuentes no convencionales de energía renovable en Colombia, con lo cual se apunta, además, el atractivo que representan los incentivos tributarios que favorecen la incursión en este mercado no explotado (Ñustes y Rivera, 2017).

\subsection{Subtemática II: modelación de incertidumbre de sistemas solares, eólicos, pequeñas centrales hidroeléctricas y vehículos eléctricos}

\subsubsection{Funciones de costo de incertidumbre para generación solar fotovoltaica, generación de energía eólica y vehículos eléctricos plug-in: valor matemático esperado y verificación por simulación de Monte Carlo.}

Los sistemas de energía eléctrica que incorporan fuentes de energía solar o eólica, o cargas controlables, como por ejemplo vehículos eléctricos, deben hacer frente a la incertidumbre sobre la disponibilidad de energía inyectada o demandada. Esto crea costos de incertidumbre a considerar en los modelos estocásticos de despacho económico.

La estimación de estos costos es importante para la correcta gestión de los recursos energéticos y la asignación precisa de la cantidad de energía disponible para el sistema. Arévalo et al. (2017b) calculan fórmulas analíticas de costos de penalización de incertidumbre para la energía solar y eólica, así como para vehículos eléctricos, a través de una formulación matemática del valor esperado.

Con el fin de obtener las funciones de costo de incertidumbre propuestas, se consideran las funciones de distribución de probabilidad -PDF por sus siglas en inglés, probability distribution function - de las fuentes primarias de energía, es decir, distribución Log-normal para irradiación solar, distribución de Rayleigh para velocidad de viento y 
distribución Normal para carga y descarga de vehículos eléctricos. La formulación analítica se verifica a través de simulaciones de Monte Carlo (Arévalo et al., 2017b).

\subsubsection{Formulación de funciones de costo de incertidumbre en pequeñas centrales hidroeléctricas dentro de una microrred.}

Molina Sánchez, Pérez Sichacá y Rivera (2017) plantean la propuesta de extender el concepto de costo de incertidumbre en la operación de sistemas de energía que contienen fuentes renovables - solar y eólica- a sistemas de potencia con penetración de pequeñas centrales hidroeléctricas $(\mathrm{PCH})$.

Para esto, se emplea la distribución de probabilidad del caudal en el que se encuentra la $\mathrm{PCH}$, lo cual posibilita el desarrollo analítico de los costos de incertidumbre en pequeñas centrales hidroeléctricas. De esta manera, se obtiene la probabilidad de potencia inyectada por la central, lo cual permite la formulación matemática de los costos por subestimar y una sobreestimación de la potencia disponible. En adición a lo anterior, la formulación analítica se valida a través de simulaciones de Monte Carlo, lo que permite la comprobación de que en ambos casos - costo de incertidumbre con la fórmula analítica y el valor esperado de la simulación de Monte Carlo- resulta el mismo costo de incertidumbre. Como complemento, se presentan los códigos que estiman el costo esperado por medio de la formulación analítica obtenida y el costo esperado mediante el valor medio de los costos de la simulación de Monte Carlo (Molina Sánchez et al., 2017). 


\title{
4. Resultados
}

\begin{abstract}
Como resultado de la aplicación de la modelación de incertidumbre propuesta - Subtemática II - en redes similares a las identificadas en la Subtemática I, se obtuvo la compilación simplificada de un bagaje invaluable de conocimientos particularmente útiles para evaluar la integración de las tecnologías de las energías renovables en cualquier sector económico. Los resultados de los estudios analíticos y de simulación de la Subtemática II se pusieron en práctica en distintos escenarios, cuyos resultados se presentan en la Subtemática III de este artículo. El procedimiento general que se empleó para la solución de las problemáticas presentadas en la Subtemática III consiste en una combinación de modelamiento analítico y simulación. Adicionalmente, el autor ha utilizado de forma extensiva técnicas de optimización analíticas y heurísticas con el fin de resolver problemas de operación y planeación de redes con renovables.
\end{abstract}

\subsection{Subtemática III: Optimización aplicada a sistemas de potencia con penetración de energía renovable}

La subtemática III se divide en tres: la primera parte corresponde a las aplicaciones de la formulación de la modelación de incertidumbre propuestas en la Subtemática II; la segunda, a aplicaciones y beneficios de la optimización heurística en la planeación del sistema; y la tercera, a la estimación de parámetros de modelos que involucren elementos de energía renovable. 


\subsubsection{Subtemática III-A: Aplicaciones de la modelación de incertidumbre.}

\subsubsection{Aplicación de costos de incertidumbre analíticos de energía solar, eólica y vehículos eléctricos en el despacho óptimo de potencia.}

Bajo la premisa de la importancia que tienen en la actualidad las fuentes de energía renovables para contrarrestar el impacto ambiental generado por las fuentes de energía tradicional y las distintas actividades humanas, Arévalo et al. (2017a) consideran la introducción de la incertidumbre en los sistemas eléctricos de potencia que incorporan recursos renovables y vehículos eléctricos, debido a la estocasticidad de los recursos primarios y los patrones de conducción de los vehículos.

Para tales incertidumbres, se proponen costos al incorporar la variabilidad de potencia inyectada de las principales fuentes de energía renovable — solar y eólica - y potencia consumida — vehículos eléctricos-. Así mismo, se considera la variabilidad a través de las distribuciones de probabilidad de las fuentes primarias de la energía renovable —irradiación solar y velocidad del viento-.

Los costos de incertidumbre requieren de modelos probabilísticos de las fuentes primarias de generación renovable. Esta investigación plantea modelos probabilísticos en ubicaciones geográficas del territorio colombiano para generación solar fotovoltaica y generación eólica. El principal aporte de este estudio es la aplicación de los costos analíticos de incertidumbre, los cuales permiten modificar la función de costos de un despacho económico tradicional. En adición, se incorpora una propuesta de solución del problema a través de un método de optimización heurística de despacho óptimo de potencia activa y reactiva. El estudio concluye con la comparación de los costos de operación del sistema con y sin la incorporación de energías renovables (Arévalo et al., 2017a). 


\subsubsection{Despacho económico en microrredes con penetración de} energía renovable usando algoritmo de punto interior y restricciones lineales.

Arango et al. (2017) muestran en este trabajo simulaciones de posibles despachos económicos para un sistema con penetración de energías renovables cuando existen variaciones de la velocidad del viento y de radiación solar en diferentes horas del día.

El desarrollo de estas simulaciones se llevó a cabo a través de la prueba y la validación de una metodología que permitiera la minimización del costo total del sistema a partir del uso del método de punto interior utilizado por la función fmincon de Matlab. El aporte más significativo radica en la propuesta de una adaptación de las restricciones del sistema de potencia a la sintaxis de la función, lo cual requiere que estas restricciones sean lineales (Arango et al., 2017).

\subsubsection{Modelado de despacho con la incorporación de componentes de maniobra, energía eólica y penetración de vehículos eléctricos al usar técnicas heurísticas.}

Rivera y Romero (2018) presentan la implementación de una formulación óptima de despacho de potencia activa y reactiva al considerar la integración de energías renovables y redes inteligentes.

La formulación puede considerar las incertidumbres de la inyección de potencia de los generadores eólicos y de los vehículos eléctricos enchufables (PEV) a través de distribuciones de probabilidad de la velocidad del viento y el comportamiento carga/descarga de PEV. El objetivo es minimizar los costos de generación dados por una expectativa matemática, mientras se satisfacen las restricciones. Dichas restricciones se relacionan con el balance de potencia nodal, la capacidad de potencia y los límites de voltaje de los generadores y PEV, el estado posible de los componentes de maniobra, las tensiones nodales de carga y el flujo máximo de potencia de las ramas para condiciones de contingencia. 
Las restricciones se presentan de acuerdo con el manejo que se les dan dentro del algoritmo de solución. De igual manera, las variables de decisión se dividen en variables continuas - relacionadas con puntos de consigna de generadores y PEV - y variables relacionadas con el estado de los componentes de maniobra. En este caso, las posiciones son variables discretas - relacionadas con los cambiadores de toma en carga ajustables por pasos - y variables binarias — relacionadas con dispositivos de compensación de derivación conmutable-.

En general, se utilizan cuatro algoritmos heurísticos y se presenta un análisis comparativo. El modelo de despacho propuesto se demuestra por el sistema de prueba IEEE 118-bus.

\subsubsection{Control de despacho distribuido en microrredes en consideración a las pérdidas y el control distribuido, basado en teoría de juegos en microrredes al considerar pérdidas de red e integración de energía renovable.}

En este estudio - que empezó como un artículo de congreso (MojicaNava, Rivera y Quijano, 2016), y después de revista (Mojica-Nava, Rivera y Quijano, 2017), se propone un algoritmo distribuido de juegos de población para resolver el problema de despacho en microrredes, con el fin de responder, de forma dinámica, a los requerimientos del sistema. Este artículo extiende el algoritmo de dinámica de replicador distribuido al aportar cuatro contribuciones principales.

Primero, se aplicó un replicador dinámico distribuido a un despacho de generación distribuida sobre una topología de comunicación en un sistema de prueba de microrred. En segundo lugar, se consideraron las pérdidas de potencia de la microrred. En tercer lugar, se propuso un algoritmo para estimar coeficientes de pérdida robusta de la red, en consideración a diferentes patrones de demanda a través de una combinación de dos algoritmos de optimización heurística. Finalmente, las incertidumbres de la energía eólica se suponen como un caso de integración 
de generación renovable. Los resultados de simulación muestran que el algoritmo de control distribuido propuesto de una microrred es capaz de integrar el problema de despacho económico con el control de frecuencia al considerar toda la topología de red.

\subsubsection{Subtemática III-B: Aplicaciones de la optimización heurística.}

\subsubsection{Optimización heurística en el diseño de parques eólicos.}

En las últimas dos décadas ha tomado importancia el problema de la optimización del costo de la energía por medio de la ubicación de turbinas en el diseño de parques eólicos. Cantor y Rivera (2016) detallan un modelo de parque eólico y destacan la importancia de su diseño óptimo, a la vez que proponen la mejor ubicación de las turbinas en un layout.

El layout se refiere a la discretización del terreno de búsqueda en una cuadrícula a través de un arreglo de coordenadas cartesianas que, posteriormente, se codifica en una cadena binaria: un uno representa una turbina a instalar en la posición indicada y un cero indica lo contrario.

Así, entonces, se exponen las diferentes metaheurísticas aplicadas para resolver dicho problema y cómo estas han arrojado mejores resultados con relación a las técnicas empíricas utilizadas tradicionalmente. Por otra parte, se introduce una metaheurística de alto nivel basada en la caza de la larva de la hormiga león -Ant Lion Optimizer - y se muestra una técnica derivada de esta investigación, que utiliza variables binarias - Binary Ant Lion Optimizer-. Se concluye con la introducción de un problema de diseño de parque eólico, con el fin de determinar el menor costo, lo cual se obtuvo gracias a la utilización del algoritmo de variables binarias con heurísticas de bajo nivel. 


\subsubsection{Mejoras de la regulación de frecuencia utilizando el aumento de inercia de microrredes interconectadas.}

En este trabajo se analiza un posible servicio auxiliar que se podría obtener cuando, en un sistema de potencia, se encuentran múltiples microrredes (Toro, Rivera y Mojica-Nava, 2017). Ante perturbaciones, las microrredes pueden cooperar entre ellas, de manera que logran así aumentar la inercia y, por ende, ser robustas ante cambios de generación y demanda propias de la generación renovable y de las redes inteligentes.

\subsubsection{Regulación de frecuencia en sistemas de potencia que integran fuentes de energías eólicas mediante un controlador PI e imitación de inercial.}

Este artículo presenta el estudio y el análisis de un esquema de control de regulación de frecuencia implementado en un sistema de potencia que integra fuentes de energías renovables (Gómez y Rivera, 2017).

El objetivo perseguido fue alcanzar una mejora de la confiabilidad y de la estabilidad de la red ante perturbaciones propias de las energías renovables. Inicialmente, se simuló la respuesta dinámica del sistema de potencia ante disturbios repentinos y de cambio continuo, a fin de observar las desviaciones de frecuencia. De la misma forma, se implementó el bloque de control en el lazo de potencia adicional para emular la «imitación inercial» $y$, de esta manera, ralentizar la respuesta del sistema. Posteriormente, se procedió a optimizar los parámetros de control a través de algoritmos de optimización heurística con el propósito de minimizar el error de la señal de frecuencia.

\subsubsection{Vehículos eléctricos como controladores de frecuencia en una microrred}

El vehículo eléctrico, hoy en día, juega un papel muy importante en el progreso de la humanidad en muchos aspectos, ya que ofrece mejorías en movilidad, medio ambiente y tecnologías, entre otros. Gracias a sus 
atributos, resulta conveniente añadir la búsqueda de ventajas tecnológicas para su utilización en ingeniería. A partir de esto, surge la implementación de sistemas como «Vehicle To Grid» (V2G), el cual contribuye a aprovechar las características eléctricas de los vehículos para ayudar a minimizar fallas y mejorar el servicio de la red eléctrica.

El estudio elaborado por Tirado y Rivera (2018) se orienta a analizar el sistema V2G como un control de frecuencia en la red, al tener en cuenta las perturbaciones en energías renovables, mediante simulaciones de un sistema ya establecido en el software Matlab. Este permite comprobar cómo las perturbaciones de las energías renovables afectan la frecuencia de toda la red y de qué manera la implementación de una flota de vehículos eléctricos ayuda a controlar estas fluctuaciones en frecuencia.

\subsubsection{Subtemática III-C: Estimación de parámetros de modelos que involucren elementos de energía renovable.}

\subsubsection{Estimación heurística de parámetros en modelos de alta frecuencia de máquinas de inducción para simulación de corrientes de rodamientos.}

Una de las principales desventajas en la introducción de dispositivos electrónicos de potencia en el control de máquinas eléctricas es el aumento de interferencias electromagnéticas (EMI).

En este estudio, Mejía, Rodríguez, Rivera y Rosero (2016) proponen una herramienta de estimación de parámetros sencilla y flexible para modelos de alta frecuencia (HF) que permite una comprensión completa de los fenómenos de EMI. La herramienta de estimación presentada utiliza la optimización heurística a fin de mejorar la correspondencia entre mediciones y simulaciones de las impedancias de modo común (CM) y diferencial (DM). La función objetivo de la formulación propuesta se establece con el objetivo de minimizar el error entre las impedancias medidas y simuladas de una máquina de inducción - tipo de máquina 
utilizada en las turbinas eólicas—- en la que se consideran diferentes tipos de errores.

De esta manera, el enfoque propuesto utiliza un algoritmo capaz de utilizar cualquier estrategia de búsqueda heurística, manejar diferentes tipos de modelos de HF y utilizar distintos métodos de comparación entre mediciones y simulaciones. Finalmente, como aplicación industrial, la estimación de parámetros se utiliza en un modelo capaz de simular corrientes de rodamiento.

\subsubsection{Coeficientes robustos de pérdidas: aplicación a sistemas de potencia con energía solar y eólica.}

En este artículo se realizan simulaciones de Monte Carlo que consideran la generación solar y eólica (Arango, Urrego y Rivera, 2018). En los posibles escenarios se propone evaluar las pérdidas a través de coeficientes de pérdidas sin tener que desarrollar un flujo de potencia para cada escenario.

A fin de determinar estos coeficientes, se utiliza optimización heurística que compara las pérdidas del sistema para variaciones de frecuencia y demanda, de modo tal que soluciona el flujo de potencia y minimiza el error entre estas pérdidas y las que se obtienen con coeficientes de pérdidas.

\subsubsection{Estimación de coeficientes del modelo de alta frecuencia de transformadores usando optimización heurística y criterios de comparación de señales y laboratorio virtual de transformadores monofásicos.}

En estos trabajos se estiman los parámetros de los modelos de transformadores, específicamente el modelo de alta frecuencia (Álvarez y Rivera, 2018), y de estado estable (Ramírez y Rivera, 2016; 2017). Cuando en el sistema de potencia existe alta penetración de energías renovables, existirán componentes de alta frecuencia debido a los conversores de corriente 
alterna a directa $(\mathrm{AC} / \mathrm{DC})$, directa a directa (DC/DC), e inversores (DC/ AC) que operan con electrónica de potencia. Así, se propone un método de estimación de parámetros que minimiza el error entre las señales simuladas del modelo y las señales obtenidas experimentalmente.

\section{Conclusiones y recomendaciones}

L

as conclusiones más significativas de este estudio se sintetizan en la subtemáticas I y II se refieren a los aspectos innovadores, y la Subtemática III a la aplicabilidad y los beneficios de la propuesta.

\subsection{Aspectos innovadores}

\subsubsection{Subtemática I.}

América Latina debe considerarse un territorio prometedor para la penetración de fuentes de energía renovables no convencionales. De igual forma, es importante acotar que, a nivel mundial, los países en desarrollo llevan a cabo investigaciones y evidencian avances en la utilización de microrredes y generación distribuida, así como en el modelado de sistemas que involucran diferentes fuentes renovables y estrategias de control aplicadas a diversos escenarios.

Particularmente, en el caso de Colombia, resulta imperante la preparación de planes de inversión por parte de los diferentes actores del sector eléctrico en los que se contemple alta penetración de generación distribuida, redes inteligentes, microrredes y parques de generación renovable no convencionales. Es evidente el panorama favorable que exhibe el país para la inversión de fuentes no convencionales de energía renovable. 


\subsubsection{Subtemática II.}

La aplicación de costos de incertidumbre analíticos de energía solar, eólica y en vehículos eléctricos en el despacho óptimo de potencia, es un modelo que representa una posible alternativa de toma de decisiones a considerar por los operadores de los sistemas de potencia, si se tienen en cuenta las incertidumbres de las fuentes primarias de la energía renovable.

La importancia de la formulación de funciones de costo de incertidumbre en pequeñas centrales hidroeléctricas dentro de una microrred radica en la alta penetración de energías renovables y la generación distribuida que tendrán los sistemas de potencia en un futuro cercano.

Dada la incertidumbre causada por la incorporación de fuentes de energía renovables, los operadores del sistema eléctrico deben disponer de herramientas o metodologías que minimicen el riesgo asociado a ellas. Las formulaciones matemáticas resultan particularmente útiles, ya que se pueden incorporar en las técnicas de optimización del sistema de potencia a fin de obtener modelos estocásticos de despacho económico.

Es imprescindible la evaluación de la viabilidad económica de los generadores basados en energías renovables a través de la modelación matemática; todo esto, con el fin de asegurar la estabilidad en el mercado eléctrico.

\subsection{Aplicabilidad y beneficios}

\subsubsection{Subtemática III}

El abordaje de problemas de optimización a través de técnicas heurísticas y metaheurísticas arroja mejores resultados que los obtenidos por medio de técnicas empíricas. Así mismo, el uso de algoritmos basados 
en el manejo de variables binarias permite llegar a respuestas de mejor calidad.

El uso del algoritmo de punto interior y de restricciones lineales para la estimación del despacho económico en microrredes con penetración de energía renovable, contribuye a enriquecer las simulaciones necesarias para programar un despacho económico óptimo cuando hay una alta incertidumbre de las inyecciones de energía, lo cual permite modelar con ello una numerosa cantidad de escenarios posibles.

La incorporación de nuevos elementos en las redes eléctricas ha introducido varios desafíos a la óptima operación del sistema de energía. Una parte importante de esta operación es el despacho económico. El objetivo de la optimización es minimizar las expectativas matemáticas de los costos de generación, al tiempo que satisface las restricciones para las condiciones de no contingencia y contingencia.

El software de ingeniería Matlab es una herramienta computacional que permite modelar, diseñar y verificar una gran variedad de sistemas dinámicos. A partir de Simulink y los demás productos de MathWorks, fue posible desarrollar y optimizar el control de las respuestas con el fin de estudiar el comportamiento de sistemas eléctricos bajo múltiples condiciones de operación.

\section{Referencias}

Álvarez, D.; Rivera, S. (2018). Parameter estimation of circuit model for distributiosn transformers. En Application of Modern Heuristic Optimization Techniques in Power and Energy Systems (573-590). Wiley.

Arango, D.; Urrego, R.; Rivera, S. (2017). Despacho económico en microrredes con penetración de energía renovable usando algoritmo de punto 
interior y restricciones lineales. Revista Ingeniería y Ciencia, 13(25), 193-222. DOI: https://doi.org/10.17230/ingciencia.13.25.5

Arango, D.; Urrego, R.; Rivera, S. (2018). Robust loss coefficients: application to power systems with solar and wind energy. International Journal of Power and Energy Conversion. DOI: https://doi. org/10.1504/IJPEC.2018.094957

Arévalo, J. C.; Santos, F.; Rivera, S. (2017a). Aplicación de costos de incertidumbre analíticos de energía solar, eólica y vehículos eléctricos en el despacho óptimo de potencia. Revista Ingeniería, 22(3), 324346. DOI: https://doi.org/10.14483/23448393.11673

Arévalo, J. C.; Santos, F.; Rivera, S. (2017b). Uncertainty cost functions for solar photovoltaic generation, wind energy generation, and plugin electric vehicles: mathematical expected value and verification by Monte Carlo Simulation. International Journal of Power and Energy Conversion. Recuperado de http://bit.ly/33JhpCl

Cantor, J.; Rivera, S. (2016). Optimización heurística en el diseño de parques eólicos. Ingeniería y Región, 16(1), 9-24. DOI: https://doi. org/10.25054/22161325.1281

Gómez, N.; Rivera, S. (2017). Regulación de frecuencia en sistemas de potencia que integran fuentes de energías eólicas mediante un controlador PI e imitación de inercia. Revista Ingeniería Solidaria, 13(23), 7-28. DOI: https://doi.org/10.16925/in.v23i13.1981

Mejía, W.; Rodríguez, D.; Rivera, S.; Rosero, J. (2016). Heuristic estimation of parameters in high-frequency models of induction motors for bearing currents simulation. International Review of Automatic Control (IREACO), 9(6), 355-364. DOI: https://doi.org/10.15866/ireaco. v9i6.9827

Mojica-Nava, E.; Rivera, S.; Quijano, N. (2016). Distributed dispatch control in microgrids with network losses. En 2016 IEEE Conference 
on Control Applications (CCA) (pp. 285-290). Buenos Aires. DOI: https://doi.org/10.1109/CCA.2016.7587850

Mojica-Nava, E.; Rivera, S.; Quijano, N. (2017). Game-theoretic dispatch control in microgrids considering network losses and renewable DER integration. IET Generation, Transmission and Distribution, 11(6), 1583-1590. DOI: https://doi.org/10.1049/iet-gtd.2016.1486

Molina Sánchez, F. S.; Pérez Sichacá, S. D.; Rivera, S. (2017). Formulación de funciones de costo de incertidumbre en pequeñas centrales hidroeléctricas dentro de una microgrid. Revista de Ingenierías USBMED, 8(1), 29-36. DOI: https://doi.org/10.21500/20275846.2683

Ñustes, W.; Rivera, S. (2017). Colombia: territorio de inversión en fuentes no convencionales de energía renovable para la generación eléctrica. Revista Ingeniería Investigación y Desarrollo, 17(1), 37-48. DOI: https://doi.org/10.19053/1900771X.v17.n1.2017.5954

Ramírez, J.; Rivera, S. (2016). Laboratorio virtual de transformadores monofásicos. Dirección Nacional de Innovación Académica, Universidad Nacional de Colombia. Recuperado de www.virtual.unal.edu. co/innovaciones/lvtm

Ramírez, J.; Rivera, S. (2017). Aplicación del ciclo de vida y el análisis estructurado en el desarrollo de un laboratorio virtual de transformadores monofásicos. Revista Educación en Ingeniería, 12(23), 43-48. DOI: https://doi.org/10.26507/rei.v12n23.732

Rivera, S.; León, M. (2017). Microgrids in the United States. En Clean energy microgrids (pp. 283-312). IET Institution of Engineering and Technology. DOI: https://doi.org/10.1049/PBPO090E_ch9

Rivera, S.; Ñustes, W.; León, M.; Rodríguez, J. (2017). Microgrids in developing countries. En Clean energy microgrids (313-348). IET Institution of Engineering and Technology. DOI: https://doi.org/10.1049/ PBPO090E_ch10 
Rivera, S.; Romero, A. (2018). Dispatch modeling incorporating maneuver components, wind power and electric vehicles penetration, using heuristic techniques. En Application of modern heuristic optimization techniques in power and energy systems. Wiley.

Rivera, S.; Valencia-Zuluaga, T. (2017). Microgrids in Europe. En Clean energy microgrids. (259-282). IET Institution of Engineering and Technology. DOI: https://doi.org/10.1049/PBPO090E_ch8

Tirado, S.; Rivera, S. (2018). Vehículos eléctricos como controladores de frecuencia en una microrred. Journal de Ciencia e Ingeniería, 10(1), 6-17.

Toro, B. V.; Rivera, S.; Mojica-Nava, E. (2017). Mejoras de la regulación de frecuencia utilizando el aumento de inercia de microrredes interconectadas. UIS Ingenierías, 16(2), 33-40. DOI: https://doi. org/10.18273/revuin.v16n2-2017003

Valencia-Zuluaga, T.; Rivera, S.; Sánchez-Ortiz, N. (2017). Potencial en el uso de la consulta de patentes para determinar el estado de la técnica. Análisis de microrredes con energías renovables. Revista Ingeniería Investigación y Desarrollo, 17(2), 16-22. DOI: https://doi. org/10.19053/1900771X.v17.n2.2017.7180

Vargas, A.; Saavedra, O.; Samper, M.; Rivera, S.; Rodríguez, R. (2016). Latin American energy markets: investment opportunities in nonconventional renewables. IEEE Power and Energy Magazine, 14(5), 3847. DOI: https://doi.org/10.1109/MPE.2016.2573862 